\title{
Safety of withholding anticoagulation in pregnant women with suspected deep vein thrombosis following negative serial compression ultrasound and iliac vein imaging
}

\author{
Wee-Shian Chan MD, Frederick A. Spencer MD, Agnes Y. Y. Lee MD, Sanjeev Chunilal MB ChB, \\ James D. Douketis MD, Marc Rodger MD, Jeffrey S. Ginsberg MD
}

\begin{abstract}
Competing interests: Sanjeev Chunilal has received consultancy fees from Bayer, travel fees from Amgen and payment for lectures from Sanofi, and he holds a study grant from Covidien. Marc Rodger holds grants from Pfizer, Boehringer Ingelheim, GTC Therapeutics, bioMérieux and LEO Pharma. James Douketis has served as a consultant for Boehringer Ingelheim, Bayer, BristolMyers Squibb and Pfizer. No competing interests declared by Wee-Shian Chan, Frederick Spencer, Agnes Lee and Jeffrey Ginsberg.
\end{abstract}

This article has been peer reviewed.

Correspondence to: Wee-Shian Chan, weeshian.chan@cw.bc.ca

CMAJ 2013. DOI:10.1503 /cmaj.120895

\begin{abstract}
Background: Compression ultrasonography performed serially over a 7-day period is recommended for the diagnosis of deep vein thrombosis in symptomatic pregnant women, but whether this approach is safe is unknown. We evaluated the safety of withholding anticoagulation from pregnant women with suspected deep vein thrombosis following negative serial compression ultrasonography and iliac vein imaging.
\end{abstract}

Methods: Consecutive pregnant women who presented with suspected deep vein thrombosis underwent compression ultrasonography and Doppler imaging of the iliac vein of the symptomatic leg(s). Women whose initial test results were negative underwent serial testing on 2 occasions over the next 7 days. Women not diagnosed with deep vein thrombosis were followed for a minimum of 3 months for the development of symptomatic deep vein thrombosis or pulmonary embolism.

Results: In total, 221 pregnant women presented with suspected deep vein thrombosis.
Deep vein thrombosis was diagnosed in 16 (7.2\%) women by initial compression ultrasonography and Doppler studies; none were identified as having deep vein thrombosis on serial testing. One patient with normal serial testing had a pulmonary embolism diagnosed 7 weeks later. The overall prevalence of deep vein thrombosis was $7.7 \%$ (17/221); of these, $65 \%(11 / 17)$ of cases were isolated to the iliofemoral veins and $12 \%(2 / 17)$ were isolated iliac deep vein thromboses. The incidence of venous thromboembolism during follow-up was $0.49 \%$ (95\% confidence interval [CI] $0.09 \%-2.71 \%$ ). The sensitivity of serial compression ultrasonography with Doppler imaging was $94.1 \%(95 \% \mathrm{Cl} 69.2 \%-99.7 \%)$, the negative predictive value was $99.5 \%(95 \% \mathrm{Cl}$ $96.9 \%-100 \%)$, and the negative likelihood ratio was 0.068 (95\% Cl 0.01-0.39).

Interpretation: Serial compression ultrasonography with Doppler imaging of the iliac vein performed over a 7-day period excludes deep-vein thrombosis in symptomatic pregnant women.
$\mathrm{O}$ ver the last 2 decades, venous compression ultrasonography has become the imaging test of choice for diagnosing deep vein thrombosis in the lower extremities of men and nonpregnant women. ${ }^{1-4}$ Although this test is highly sensitive (about 97\%) for deep vein thrombosis involving the femoral and popliteal veins, compression ultrasonography is less sensitive for the detection of isolated deep vein thrombosis in the calf. ${ }^{5}$ Because proximal propagation of isolated calf deep vein thrombosis occurs in about $20 \%$ of cases, serial compression ultrasonography performed over a 7-day period is recommended to definitely exclude such thromboses if the results of the initial compression ultrasound are negative. ${ }^{6}$

The use of serial compression ultrasonography in symptomatic men and nonpregnant women has been validated in prospective studies, ${ }^{1,7}$ suggest- ing that withholding anticoagulation from symptomatic patients whose serial compression ultrasound results are negative is safe, with less than $2 \%$ of patients subsequently being diagnosed with deep vein thrombosis. ${ }^{1,7,8}$ Although the use of serial compression ultrasonography has not been validated in pregnant women, this strategy is also advocated for symptomatic pregnant women.'

The appeal of using compression ultrasonography for diagnosing deep vein thrombosis in pregnant women is obvious: it is noninvasive, widely available and does not expose the fetus to ionizing radiation. However, generalizing results from studies involving men and nonpregnant women to pregnant women is problematic because of differences in clinical presentation and anatomic distribution of deep vein thromboses. ${ }^{10}$ Compared with men and nonpregnant women, pregnant women 
more often present with very proximal deep vein thrombosis (including isolated iliac vein deep vein thrombosis); isolated distal calf deep vein thromboses are infrequent. ${ }^{10}$ In a recent review of the literature, we found that $62 \%$ of all deep vein thromboses in symptomatic pregnant women were in the iliofemoral veins, $17 \%$ were in the iliac vein alone, and $6 \%$ were in the calf veins. ${ }^{10} \mathrm{In}$ contrast, in the general population, more than $80 \%$ of deep vein thromboses involved calf veins, and iliofemoral deep vein thromboses or isolated iliac veins are uncommon $(<5 \%) .^{1.4}$

Physiologic changes associated with pregnancy might affect blood flow patterns and normal compressibility of the proximal veins, thereby affecting the diagnostic accuracy of compression ultrasonograpy. This technique cannot be used to detect isolated deep vein thromboses in the iliac vein; these veins are not compressible because of their intrapelvic location. Whether Doppler studies are sensitive for detecting deep vein thromboses in these high proximal veins (i.e., iliac veins) has not been well studied, but data suggest that this method of detection compares favourably to compression ultrasonography in men and nonpregnant women for proximal deep vein thromboses. ${ }^{11}$ The use of Doppler imaging in pregnant women for the purpose of detecting iliac vein deep vein thromboses has been reported in the literature, ${ }^{12,13}$ but it has not been adequately evaluated.

Currently, the standard practice of diagnosing deep vein thrombosis in symptomatic pregnant women is by compression ultrasonography. If the results of the compression ultrasound are negative, Doppler imaging of the iliac vein (with or without vagal manoeuvres) is recommended, particularly for women with a high clinical probability of deep vein thrombosis in the iliac vein., ${ }^{9,12,13}$ This diagnostic approach is advocated despite the absence of any prospective studies validating its use. In this study, we evaluated the diagnostic accuracy of serial compression ultrasonography and Doppler imaging of the iliac veins over a 7-day period among symptomatic pregnant women.

\section{Methods}

This prospective cohort study involved consecutive pregnant women referred for investigation of suspected deep vein thrombosis. Women were recruited between August 2002 and September 2010 from 4 Canadian centres with a dedicated thrombosis service: Sunnybrook Health Sciences Centre and Women's College Hospital, Toronto; McMaster University Medical Centre and the Juravinski Hospital, Hamilton; St. Joseph's
Healthcare Hamilton; and the Ottawa Hospital (Civic and General campuses), Ottawa, Ontario. Patients were initially identified by their primary care physicians as having clinically suspected deep vein thrombosis and were referred for investigation. After written informed consent was obtained, we collected baseline information, and the women underwent compression ultrasonography and Doppler imaging.

We included women with a suspected first episode of deep vein thrombosis. We excluded women who met 1 or more of the following criteria: received any anticoagulant therapy for more than 48 hours before presentation; had a suspected pulmonary embolism (these patients required other diagnostic imaging); had concomitant severe maternal, cardiac or pulmonary disease; were unable or unwilling to return for follow-up; or did not provide consent.

Ethics approval was obtained at all participating institutions.

\section{Ultrasound examination}

The diagnostic algorithm for the study is shown in Figure 1. Ultrasound examination of the symp-

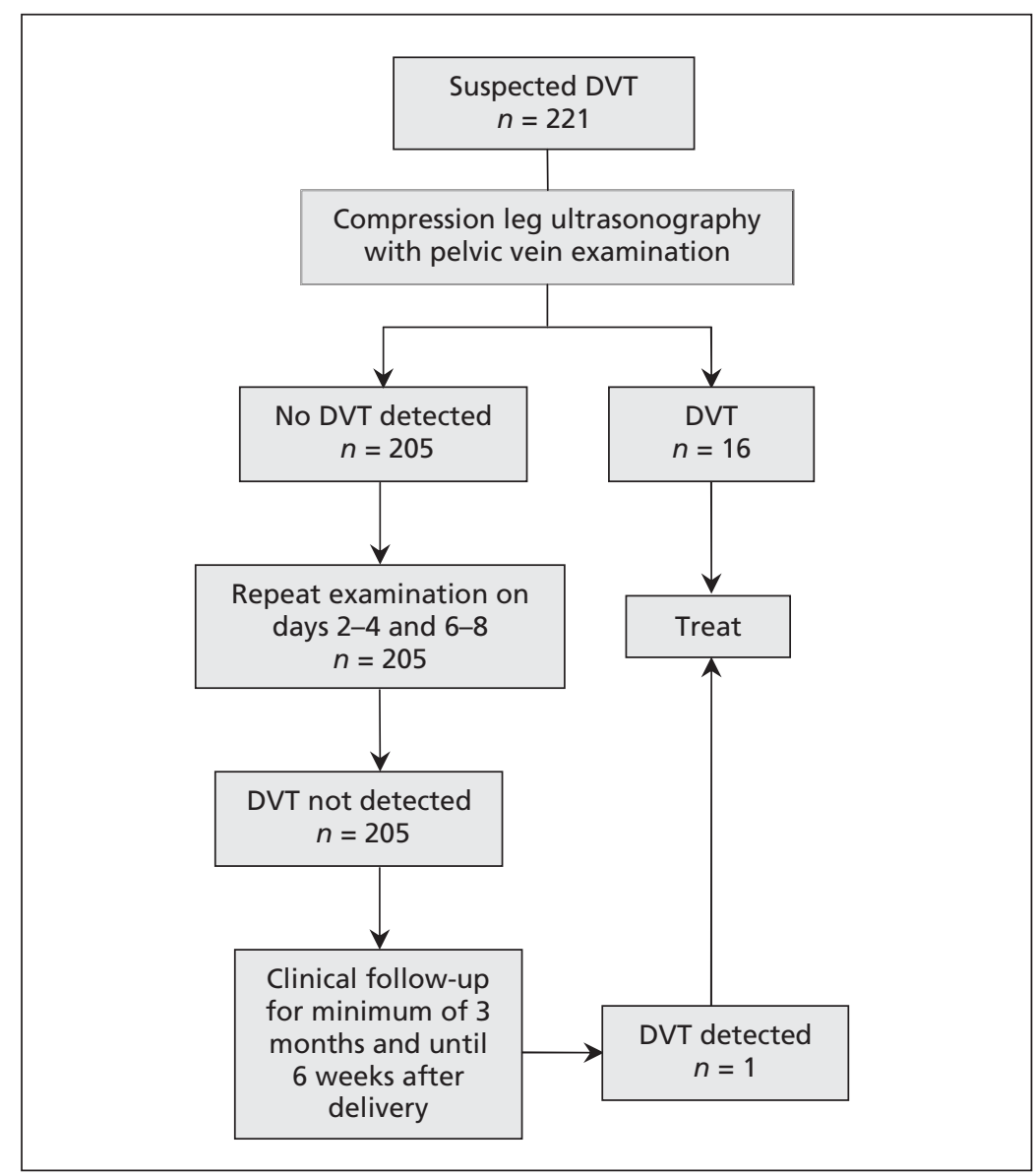

Figure 1: Diagnostic algorithm for antepartum women with suspected deep venous thrombosis (DVT) using serial compression ultrasonography and doppler imaging of the iliac vein. 
tomatic leg(s) was performed by compression of the proximal veins. Compression manoeuvres of the symptomatic leg were done at $1-\mathrm{cm}$ intervals along the length of the femoral vein (from the inguinal ligament) and popliteal veins to the level of the calf vein trifurcation.

The ipsilateral external iliac vein was also imaged in all patients. Any intraluminal echogenic masses consistent with a thrombus were noted on B-mode imaging, and spontaneous venous flow was assessed by use of Doppler interrogation. Absent or sluggish flow was further evaluated with augmentation manoeuvres.

Deep vein thrombosis was diagnosed based on noncompressibility at any 2 contiguous segments of the femoral or popliteal vein. Deep vein thrombosis was also diagnosed in the iliac veins by the absence of flow within the iliac vein and/or the presence of a visible thrombus by Doppler imaging. We excluded deep vein thrombosis based on full compressibility of the femoral and popliteal veins and normal Doppler imaging of the iliac veins.

Imaging was performed by trained technicians, and the images were reviewed by local radiologists. In all cases, abnormal findings were confirmed by the local radiologist.

If initial results were negative for deep vein

Table 1: Characteristics of 221 pregnant women who presented with suspected deep venous thrombosis

\begin{tabular}{|c|c|}
\hline Characteristic & Number $(\%)^{*}$ \\
\hline Age, yr, mean (range) & $32(20-47)$ \\
\hline \multicolumn{2}{|l|}{ Ethnic background } \\
\hline White & 189 (85.5) \\
\hline African & $6 \quad(2.7)$ \\
\hline Asian or Pacific Islander & $21 \quad(9.5)$ \\
\hline Hispanic & $3 \quad(1.4)$ \\
\hline Mixed & $2 \quad(0.9)$ \\
\hline Nulliparous & 114 (51.6) \\
\hline Singleton & 191 (86.4) \\
\hline Multiples & $30 \quad(13.6)$ \\
\hline \multicolumn{2}{|l|}{ Trimester } \\
\hline First ( $\leq 12 \mathrm{wk})$ & $15 \quad(6.8)$ \\
\hline Second (> 12-28 wk) & $82 \quad(37.1)$ \\
\hline Third (> 28 wk) & $124 \quad(56.1)$ \\
\hline \multicolumn{2}{|l|}{ Symptomatic leg } \\
\hline Left & $97 \quad(43.9)$ \\
\hline Right & $91 \quad(41.2)$ \\
\hline Bilateral & 33 (14.9) \\
\hline Presence of risk factors $t$ & $80 \quad(36.2)$ \\
\hline $\begin{array}{l}\text { *Unless otherwise stated } \\
\text { †Malignant disease, trauma, far } \\
\text { thrombophilia, prolonged bed }\end{array}$ & e techniques, known \\
\hline
\end{tabular}

thrombosis (day 0), anticoagulant therapy was withheld and repeat testing was conducted after 2-4 days and 6-8 days. If the subsequent test results were positive, anticoagulant therapy was initiated.

\section{Clinical follow-up}

Patients whose serial test results did not show deep vein thrombosis were followed clinically for a minimum of 3 months and at least 6 weeks after delivery for symptoms of deep vein thrombosis or pulmonary embolism. At the end of the followup period, patients were contacted by telephone or seen in person to verify that no symptomatic events had occurred. If signs or symptoms of possible deep vein thrombosis developed within the follow-up period, patients underwent repeat serial testing with compression ultrasonography and Doppler studies. If signs or symptoms of possible pulmonary embolism developed during followup, patients underwent ventilation-perfusion scanning. Pulmonary embolism was diagnosed in cases where the ventilation-perfusion scan showed a high probability. If the results of the perfusion scan were normal, pulmonary embolism was excluded. If the ventilation-perfusion scan was nondiagnostic (low or indeterminate probability), the patient underwent testing for deep vein thrombosis, spiral computed tomography or both, depending on local practice.

\section{Statistical analysis}

Patient characteristics are presented as proportions. We classified patients as having deep vein thrombosis if deep vein thrombosis had been diagnosed based on the results of compression ultrasonography and Doppler imaging of the pelvic veins during the initial or serial testing, or if deep vein thrombosis or pulmonary embolism were diagnosed on follow-up during investigation of symptoms.

We present the rate of venous thromboembolism on follow-up as a proportion with $95 \%$ confidence intervals (CIs). We described the test characteristics of our overall diagnostic strategy by presenting sensitivity, negative predictive value (the proportion of patients with a negative result who did not have deep vein thrombosis) and negative likelihood ratio (the probability of a patient with deep vein thrombosis having negative test results divided by the probability of a patient without deep vein thrombosis having negative results) with $95 \%$ CIs.

\section{Results}

Over the 8-year study period, 221 consecutive pregnant women presented with symptoms sug- 
gestive of deep vein thrombosis (Table 1). All eligible patients were recruited into the study: 90 women from Sunnybrook Health Sciences Centre and Women's College Hospital; 65 from McMaster University Medical Centre and the Juravinski Hospital; 39 from St. Joseph's Healthcare Hamilton; and 27 from the Ottawa Hospital. Of these women, $51.6 \%$ were nulliparous, $86.4 \%$ had singleton pregnancies and $56.1 \%$ presented during the third trimester. The left leg was symptomatic in $43.9 \%$ of patients and the right leg was symptomatic in $41.2 \%$ of patients. In $36.2 \%$ of patients with symptoms suggestive of deep vein thrombosis, additional risk factors (e.g., malignant disease, trauma, family history, use of assisted reproductive techniques, known thrombophilia, prolonged bed rest) were present.

Deep vein thrombosis was diagnosed in 16 women $(7.2 \%)$ by initial compression ultrasonography with Doppler imaging; these thromboses were subsequently treated with anticoagulant therapy. One of these patients, who was diagnosed as having extensive iliofemoral deep vein thrombosis at study enrolment, had been assessed 6 days earlier at a separate facility, at which time the results of a single compression ultrasonographic study were reported as negative for deep vein thrombosis (the iliac veins were not imaged). During follow-up, 6 of the 205 women who had normal serial test results later presented with symptoms of deep vein thrombosis, pulmonary embolism or both. Of these, 1 woman was diagnosed as having deep vein thrombosis and pulmonary embolism. This patient had negative bilateral serial test results when bilateral leg symptoms developed at 10 weeks' gestation. The

Table 2: Findings from serial compression ultrasonography with iliac vein visualization for 17 pregnant women with deep vein thrombosis

\begin{tabular}{|c|c|c|c|c|c|c|c|c|}
\hline Patient & CUS order* & $\begin{array}{l}\text { Side of } \\
\text { DVT }\end{array}$ & $\begin{array}{l}\text { External } \\
\quad \text { Iliac }\end{array}$ & Femoral & $\begin{array}{l}\text { Superficial } \\
\text { femoral }\end{array}$ & Popliteal & Calf & Note \\
\hline 1 & First & Left & + & + & - & - & - & \\
\hline 2 & First & Left & + & + & + & - & - & \\
\hline 3 & First & Left & + & + & + & + & - & \\
\hline 4 & First & Left & + & + & - & - & - & \\
\hline \multirow[t]{2}{*}{6} & First & Right & - & + & - & & - & \multirow{2}{*}{$\begin{array}{l}2.1-\mathrm{cm} \text { nonocclusive thrombus } \\
\text { found in the right common } \\
\text { femoral vein on day } 0 \text {. } \\
\text { Therapeutic anticoagulation was } \\
\text { initiated on day } 0 \text {, and CUS was } \\
\text { repeated on day } 5\end{array}$} \\
\hline & Second & & + & + & + & - & - & \\
\hline 10 & First & Left & + & + & + & + & - & \\
\hline 11 & First & Left & - & + & - & - & - & \\
\hline 12 & First & Left & + & + & + & - & - & \\
\hline 13 & First & Left & + & + & - & - & - & $\begin{array}{l}\text { CUS at another facility } 6 \text { days } \\
\text { earlier was negative for DVT }\end{array}$ \\
\hline 14 & First & Right & - & + & + & + & - & \\
\hline \multicolumn{9}{|l|}{15} \\
\hline \multirow[t]{2}{*}{$\begin{array}{l}\text { Initial } \\
\text { presentation }\end{array}$} & First & - & - & - & - & - & - & $\begin{array}{l}\text { Bilateral leg CUS was done on } \\
\text { initial presentation }\end{array}$ \\
\hline & Second & - & - & - & - & - & - & \\
\hline
\end{tabular}


fetus was miscarried (attributed to a massive fibroid) 7 weeks after initial presentation, and the patient was diagnosed with deep vein thrombosis and pulmonary embolism (high probability on a ventilation-perfusion scan) 2 days after the miscarriage.

The proportion of venous thromboembolism during follow-up was $0.49 \%$ (95\% CI $0.09 \%$ $2.71 \%$ ). The overall prevalence of deep vein thrombosis in our study cohort was $7.7 \%$ (95\% CI $4.9 \%-12.0 \%$ ). Deep vein thrombosis was diagnosed in the left leg in $82 \%$ of cases and in the right leg in the $18 \%$. None of the women experienced bilateral deep vein thromboses. The anatomic findings of the 17 pregnant women with deep vein thrombosis are shown in Table 2. The iliac or femoral veins, or both, were involved in $65 \%(11 / 17)$ of cases. Of these, 2 $(12 \%)$ cases were isolated to the iliac vein, and 4 (24\%) were isolated to the femoral vein.

The data used to calculate the test characteristics for compression ultrasonography with Doppler imaging of the iliac vein are shown in Table 3. The sensitivity of both the initial and serial compression ultrasonographic studies and Doppler imaging of the iliac vein was $94.1 \%$ (95\% CI $69.2 \%-99.7 \%$ ), the negative predictive value was $99.5 \%$ (95\% CI $96.9 \%$ $100 \%)$ and the negative likelihood ratio was 0.068 (95\% CI 0.01-0.39). Serial testing over a 7-day period did not detect any additional cases of deep vein thrombosis or pulmonary embolism.

\section{Interpretation}

In our study, only $7.7 \%$ of pregnant women with symptoms of deep vein thrombosis had objectively confirmed diagnoses. Of these, $94 \%$ were detected at presentation by compression ultrasonography with Doppler imaging of the ipsilateral iliac vein; serial testing did not detect new thromboses. The sensitivity of serial compression ultrasonography with Doppler imaging was $94.1 \%$, and the proportion of venous thromboembolism during follow-up was $0.49 \%$. Our

\begin{tabular}{|lccc|}
\hline \multicolumn{4}{|l|}{$\begin{array}{l}\text { Table 3: Deep vein thromboses identified } \\
\text { among } 221 \text { symptomatic pregnant women by } \\
\text { serial compression ultrasonography and Doppler } \\
\text { imaging }\end{array}$} \\
\hline Test result & $\begin{array}{c}\text { Thrombosis } \\
\text { absent }\end{array}$ & $\begin{array}{c}\text { Thrombosis } \\
\text { present }\end{array}$ & Total \\
\hline Positive & 0 & 16 & 16 \\
\hline Negative & 204 & 1 & 205 \\
\hline Total & 204 & 17 & 221 \\
\hline
\end{tabular}

strategy of serial compression ultrasonography combined with Doppler imaging of the iliac veins appears to reliably exclude clinically important deep vein thrombosis.

Our study also highlights the finding that most deep vein thromboses in pregnant women occur in the high proximal veins..$^{10}$ In other words, deep vein thromboses commonly occur in the iliac vein (usually on the left side); the thrombosis can then propagate distally into the femoral vein or proximally into the inferior vena cava. This is in contrast with the natural history of deep vein thrombosis in men and nonpregnant women, in whom isolated iliac deep vein thrombosis is uncommon and most thrombi originate in the calf veins. ${ }^{6}$

Our findings of the sensitivity and specificity of serial compression ultrasonography with Doppler imaging to exclude deep vein thrombosis in pregnant women are consistent with studies involving men and nonpregnant women. ${ }^{1,5}$ Heijboer and colleagues ${ }^{1}$ reported an event rate of $1.5 \%$ (95\% CI $0.5 \%-3.3 \%$ ) among consecutive outpatients after negative serial compression ultrasonography, with calculated sensitivity of 93.3\% (95\% CI 8.5\%-97.3\%).

There are few published studies that have rigorously evaluated whole-leg examination or serial compression ultrasonography in pregnant women. The prevalence of deep vein thrombosis was reported to be $27 \%$ (of 162 pregnant and postpartum women with suspected deep vein thrombosis) in a retrospective study of the use of whole-leg examination with Doppler imaging of the iliac veins. ${ }^{14}$ Although the authors reported that a single compression ultrasonographic study appeared to rule out deep vein thrombosis, clinical follow-up was only achieved in $91 \%$ of patients, and more than $10 \%$ of patients without diagnosed deep vein thrombosis received post-test anticoagulation therapy.

In a small observational study $(n=87),{ }^{15}$ serial compression ultrasonography was performed over a 7-day period after an initial whole-leg examination (with colour Doppler of the iliac veins) to rule out deep vein thrombosis in symptomatic pregnant women. The authors reported that $34 \%$ of patients were diagnosed with deep vein thrombosis. Of the 57 patients whose initial compression ultrasonography results were negative, 55 underwent serial testing (no positive results), and only 43 completed 3-month follow-up. ${ }^{15}$

\section{Limitations}

There are several potential limitations to our study. First, the specificity of compression ultra- 
sonography to diagnose deep vein thrombosis by vein compression or direct thrombus imaging is assumed to be $100 \%$, because the reference standard (leg venography with fluoroscopy or computed-tomographic angiography) cannot ethically be performed for pregnant women to confirm deep vein thrombosis diagnosed by compression ultrasonography. Thus, we assumed that all abnormalities diagnosed represent deep vein thrombi, especially those in the pelvic veins - a site where compression manoeuvres cannot be performed.

Second, the prevalence of deep vein thrombosis in our cohort was low (as compared with the prevalence seen in studies of men and nonpregnant women $\left.{ }^{1-4}\right)$. This could exaggerate the negative predictive value (i.e., the negative predictive value is closer to $100 \%$ because the prevalence of the disease is low). Corresponding with the low prevalence, the sensitivity and likelihood ratios had wide confidence intervals. The prevalence of deep vein thrombosis in our study is lower than that reported in 2 other studies. ${ }^{14,15}$ This could be because the study by Le Gal and colleagues $^{14}$ involved both pregnant and postpartum women and because the methods used by Ratiu and colleagues ${ }^{15}$ for patient selection and recruitment are unclear. The prevalence in our study was, however, similar to that reported in a diagnostic study involving pregnant women with suspected deep vein thrombosis that used a different diagnostic modality. ${ }^{16}$ Our study reinforces the finding that nonthrombotic leg symptoms are common among pregnant patients and that diagnostic imaging is key in identifying the presence of such disease.

Third, the investigators in this study were thrombosis physicians based in secondary and tertiary referral centres. However, they also provided support services to primary obstetrical care physicians at these centres. In all participating centres, the study investigators were successful in recruiting patients from antepartum wards, physicians' offices, triage and emergency facilities. Thus, our results are generalizable to most community-based hospitals.

\section{Conclusion}

Our study highlights the importance of iliac vein visualization in symptomatic pregnant women. Because all of our cases of deep vein thrombosis were identified by initial imaging with compression ultrasonography and Doppler studies, it is unclear whether serial testing over a 7-day period is necessary. A single, complete ultrasonographic examination of the proximal venous system (including the iliac veins) in symptomatic patients appears to exclude deep venous thrombosis. However, in current practice, available expertise in pelvic vein examination in most centres is unknown, and the general awareness of the pathophysiology of lower extremity venous thrombi in pregnant women is uncertain. Current advocated use of serial testing is associated with increased costs and decreased patient compliance. Future studies will investigate new diagnostic strategies using D-dimer testing and clinical prediction rules combined with ultrasonography to improve the accuracy and reliability of noninvasive testing in excluding deep vein thrombosis in symptomatic pregnant women. ${ }^{17,18}$

Our study shows that serial compression ultrasonographic studies with iliac vein visualization performed over a 7-day period can reliably exclude deep vein thrombosis in symptomatic pregnant women and that it is likely safe to withhold anticoagulation in women with negative imaging results.

\section{References}

1. Heijboer H, Buller HR, Lensing AWA, et al. A comparison of real-time compression ultrasonography with impedance plethysmography for the diagnosis of deep vein thrombosis in symptomatic outpatients. N Engl J Med 1993;19:1365-9.

2. Cogo A, Lensing AW, Koopman MM, et al. Compression ultrasound for diagnostic management of patients with clinically suspected deep vein thrombosis: prospective cohort study. BMJ 1998;7124:17-20.

3. Cogo A, Lensing AWA, Pradoni P, et al. Distribution of thrombosis in patients with symptomatic deep vein thrombosis. Arch Intern Med 1993;153:2777-80.

4. Bernardi E, Camporese G, Büller HR., et al.; Erasmus Study Group. Serial 2-point ultrasonography plus D-dimer vs wholeleg color-coded doppler ultrasonography for diagnosing suspected symptomatic deep vein thrombosis: a randomized controlled Trial. JAMA 2008;300:1653-9.

5. Kearon C, Julian JA, Ginsberg JS, et al. Noninvasive diagnosis of deep venous thrombosis. Ann Intern Med 1998;8:663-77.

6. Lohr JM, Kerr TM, Lutter KS, et al. Lower extremity calf thrombosis: To treat or not to treat? J Vasc Surg 1991;5:618-23.

7. Wells PS, Hirsh J, Anderson DR, et al. Comparison of the accuracy of impedance plethysmography and compression ultrasonography in outpatients with clinically suspected deep vein thrombosis. A two centre paired-design prospective trial. Thromb Haemost 1995;74:1423-7.

8. Heijboer H, Cogo A, Büller HR, et al. Detection of deep vein thrombosis with impedance plethysmography and real-time compression ultrasonography in hospitalized patients. Arch Intern Med 1992;152:1901-3.

9. Chan WS, Ginsberg JS. Diagnosis of deep vein thrombosis and pulmonary embolism in pregnancy [review]. Thromb Res 2002;107:85-91.

10. Chan WS, Spencer FA, Ginsberg JS. Anatomic distribution of deep vein thrombosis in pregnancy [review]. CMAJ 2010;182:657-60.

11. Cogo A, Lensing AW, Prandoni P, et al. Comparison of real-time B-mode ultrasonography and Doppler ultrasound with contrast venography in the diagnosis of venous thrombosis in symptomatic outpatients. Thromb Haemost 1993;70:404-7.

12. Frede TE, Ruthberg BN. Sonographic demonstration of iliac venous thrombosis in the maternity patient. $J$ Ultrasound Med 1988;1:33-7.

13. Palmgren J, Kirkinen P. Venous circulation in the maternal lower limb: a Doppler study with the Valsalva maneuver. Ultrasound Obstet Gynecol 1996;8:93-7.

14. Le Gal G, Prins AM, Righini M, et al. Diagnostic value of a negative single complete compression ultrasound of the lower limbs to exclude the diagnosis of deep venous thrombosis in pregnant or postpartum women: a retrospective hospital-based study. Thromb Res 2006;118:691-7.

15. Ratiu A, Navolan D, Spatariu I, et al. Diagnostic value of a nega- 
tive single color duplex ultrasound in deep vein thrombosis suspicion during pregnancy. Rev Med Chir Soc Med Nat Iasi 2010; 114:454-6.

16. Hull RD, Raskob GE, Carter CJ. Serial impedance plethysmography in pregnant patients with clinically suspected deep vein thrombosis. Ann Intern Med 1990; 9:663-7.

17. Chan WS, Lee A, Spencer FA, et al. Predicting deep venous thrombosis in pregnancy: out in "LEFt" field? [published erratum in: Ann Intern Med 2009;151:516]. Ann Intern Med 2009;151:85-92.

18. Chan WS, Lee A, Spencer FA, et al. D-dimer testing in pregnan patients: towards determining the next "level" in the diagnosis of deep vein thrombosis. J Thromb Haemost 2010;8:1004-11.

Affiliations: From the Department of Medicine (Chan, Spencer, Douketis, Ginsberg), McMaster University, Hamilton, Ont.; the Department of Medicine (Lee), University of British Columbia, Vancouver, BC; the Department of Clinical Hematology (Chunilal), Monash University, Clayton, Victoria, Australia; the Department of Medicine (Rodger), University of Ottawa, Ottawa, Ont.
Contributors: Wee-Shian Chan designed the study, drafted and revised the manuscript, performed data collection, preparation and analysis. Frederick Spencer was involved with data analysis, manuscript revision and content development. Agnes Lee, Sanjeev Chunilal, James Douketis, Marc Rodger and Jeffrey Ginsberg were involved with study design, data analysis and manuscript revision. Wee-Shian Chan, Frederick Spencer, Agnes Lee, James Douketis, Marc Rodger and Jeffrey Ginsberg were involved in patient recruitment. All authors approved the final version of the manuscript submitted for publication

Funding: The study was funded by Heart and Stroke Ontario (grant no. 5048) between July 2002 and June 2004.

Acknowledgement: Marc Rodger was the recipient of a Heart and Stroke Foundation Career Investigator Award and holds a University of Ottawa, Faculty of Medicine, Clinical Research Chair in Venous Thrombosis and Thrombophilia. 